\title{
Analysis of free vibration of cylindrical shells on the basis of three dimensional exact elasticity theory
}

\author{
Babak Gasemzadeh $^{1 *}$, Reza Azarafza ${ }^{1}$, Yaser Sahebi ${ }^{2}$, and Asadollah Motallebi ${ }^{2}$ \\ ${ }^{1}$ Department of Mechanics, Sanandaj Branch, Islamic Azad University, Sanandaj, Iran \\ ${ }^{2}$ Department of Mechanics, Khoy Branch, Islamic Azad University, Khoy, Iran \\ babakqasemzadeh@yahoo.com
}

\begin{abstract}
In this paper we present a study of free vibration of cylindrical shells on the basis of three dimensional exact theory. The studied boundary condition is simple-simple supported boundary condition. Extensive frequency parameters have been obtained through resolving frequency equations. The results of frequency parameters have been obtained by computers programs and have been compared with the results of classical theories.
\end{abstract}

Keywords: Free vibration, Cylindrical shells, Frequencies, Exact elasticity theory

Introduction
Cylindrical shells are increasingly used in the engineering and aero space industry. Almost all the structure that are made of cylindrical shells are under dynamical loads and have vibration potential. One of most important reason of failure is Resonance phenomenon. Love (1995) and flügge (1993) are valid for small displacements used for the analysis of cylindrical shells vibration. The studies on cylindrical shells vibration on the basis of 3D elasticity theory have been done by Herr and Mirsky (1989) for infinite long cylindrical shells and by $\mathrm{Ye}$ and Soldotas (1990) for finite long cylindrical shells.

Exact elasticity theory

The theory is used for the analysis of vibration of cylindrical shells are generally classical. We can classify classical theories in two types. The first type is applied for plate stress for small displacement. The second type is a three-dimensional analysis but by using some assumption this analysis is changed to two dimensional analyses. However; in classical theories, component of transverse stress and strain are ignored. By using exact elasticity theory components of transverse stress and strain are added to cylindrical shells vibration equation.

Governing vibration equation

Fig. 1. Geometry of cylindrical shell element

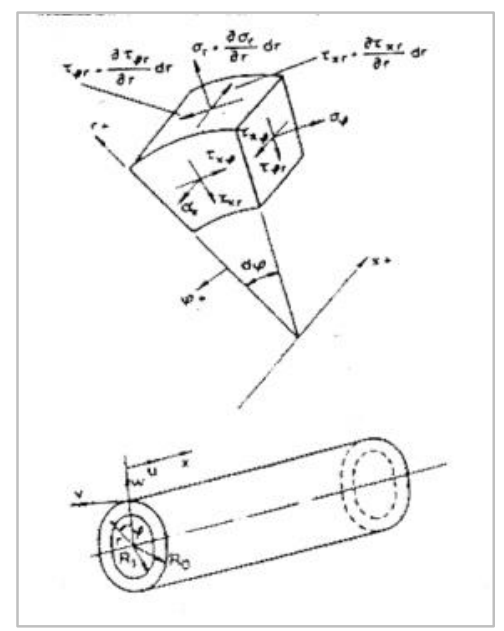

Research article

CIndian Society for Education and Environment (iSee)
Consider an element of cylindrical shell as shown in Fig. 1 by using the equilibrium conditions for the component of stress tonsures in direction of Cartesian coordinates system and in the absence of external force, we can show dynamic equations of equilibrium for the cylindrical shells element follow as:

$$
\begin{aligned}
& \frac{\partial \sigma_{x}}{\partial x}+\frac{1}{r} \frac{\partial \tau_{\varphi x}}{\partial \varphi}+\frac{\tau_{\varphi x}}{r}=\rho \frac{\partial^{2} u}{\partial t^{2}} \\
& \frac{\partial \tau_{\varphi x}}{\partial x}+\frac{1}{r} \frac{\partial \sigma_{\varphi x}}{\partial \varphi}+\frac{\partial \tau_{r \varphi x}}{\partial r}+\frac{2 \tau_{r \varphi}}{r}=\rho \frac{\partial^{2} v}{\partial t^{2}} \\
& \frac{\partial \tau_{r x}}{\partial x}+\frac{1}{r} \frac{\partial \tau_{\varphi x}}{\partial \varphi}+\frac{\partial \sigma_{r}}{\partial r}+\frac{\sigma_{r}-\sigma_{\varphi}}{r}=\rho \frac{\partial^{2} w}{\partial t^{2}}
\end{aligned}
$$

The stress $\sigma_{r}, \sigma_{\varphi}, \sigma_{x}, \tau_{x r}, \tau_{\varphi x}$ and displacements $\mathbf{u}, \mathbf{v}$ and $\mathbf{w}$ are defined in Fig. 1.

Strain and displacement relation is expressed as:

$$
\begin{aligned}
& \varepsilon_{x}=\frac{\partial u}{\partial x}, \quad \varepsilon_{\varphi}=\frac{1}{r} \frac{\partial v}{\partial \varphi}+\frac{w}{r}, \quad \varepsilon_{r}=\frac{\partial w}{\partial r} \\
& \gamma_{x \varphi}=\gamma_{\varphi x}=\frac{\partial v}{\partial x}+\frac{1}{r} \frac{\partial u}{\partial \varphi}, \quad \gamma_{x r}=\gamma_{x r}=\frac{\partial w}{\partial x}+\frac{\partial u}{\partial r} \\
& \gamma_{\varphi r}=\gamma_{r \varphi x}=+\frac{1}{r} \frac{\partial w}{\partial \varphi}+\frac{\partial v}{\partial r}-\frac{v}{r}
\end{aligned}
$$

From boundary conditions in inner and outer surface for simple supported boundary condition:

$$
\left.\sigma_{r}\right|_{r=R_{1}, R_{0}}=\left.\tau_{\varphi r}\right|_{r=R_{1}, R_{0}}=\left.\tau_{x \varphi}\right|_{r=R_{1}, R_{0}}=0
$$

$R_{1} \quad$ is inner surface radial $R_{0}$ is outer surface radial.

The following assumed solution satisfies simple supported boundary condition:

$u=U(r, \varphi) \cos \lambda * x \cos \omega t$

$v=V(r, \varphi) \sin \lambda * x \cos \omega t$

$w=W(r, \varphi) \sin \lambda * x \cos \omega t$

$\lambda *=\frac{m \pi}{L}$

(4)

We can simplify the equation by substituting Mirsky potential equation: 
$U(r, \varphi)=C \phi(r, \varphi)$

$V(r, \varphi)=\frac{1}{r} \frac{\partial \phi(r, \varphi)}{\partial \varphi}-\frac{\partial \psi(r, \varphi)}{\partial r}$

$W(r, \varphi)=\frac{\partial \phi}{\partial r}+\frac{1}{r} \frac{\partial \psi}{\partial r}$

After substituting and simplifying the relation mention above and resolving differentials equation displacements $\mathrm{u}, \mathrm{v}$ and $\mathrm{w}$ are obtained as follows:

$u=\left\{\lambda A_{m n} Z_{n}\left(q_{1} r\right)+\lambda B_{m n} \bar{Z}_{n}\left(q_{1} r\right)-\right.$

$\frac{P_{2}^{2}}{\lambda}\left[C_{m n} Z_{n}\left(q_{2} r\right)+D_{m n} \bar{Z}_{n}\left(q_{2} r\right)\right\} \cos \lambda * x \operatorname{csn} \varphi \cos \omega$

$v=\left[\frac{n}{r} A_{m n} Z_{n}\left(q_{1} r\right)+\frac{n}{r} B_{m n} \bar{Z}_{n}\left(q_{1} r\right)-\frac{n}{r} C_{m n} Z_{n}\left(q_{2} r\right)+\right.$

$\left.\frac{n}{r} D_{m n} \bar{Z}_{n}\left(q_{2} r\right)+E_{m n} \frac{d Z_{n}\left(q_{2} r\right)}{d r}+F_{m n} \frac{d \bar{Z}_{n}\left(q_{2} r\right)}{d r}\right] \sin \lambda * x \sin n \varphi \cos \omega \quad t \quad(6)$

$w=\left[A_{m n} \frac{d Z_{n}\left(q_{1} r\right)}{d r}+B_{m n} \frac{d \bar{Z}_{n}\left(q_{1} r\right)}{d r}+C_{m n} \frac{d Z_{n}\left(q_{2} r\right)}{d r}+D_{m n} \frac{d \bar{Z}_{n}\left(q_{2} r\right)}{d r}+\right.$

$\left.\frac{n}{r} E_{m n} Z_{n}\right] \sin \lambda * x \sin n \varphi \cos \omega t$

$Z_{n}, \overline{Z_{n}}$ are selected from Table. 1 arranged by Armenakas et al. (1969):

Table 1 (arranged by Armenakas et al., 1969)

\begin{tabular}{l}
\begin{tabular}{|l|l|l|l|l|}
\hline Frequency & \multicolumn{3}{|c|}{ Bessel functions $Z, \bar{Z}_{n}$} \\
\hline interval & $Z_{n}\left(q_{1} r\right)$ & $\bar{Z}_{n}\left(q_{1} r\right)$ & $Z_{n}\left(q_{2} r\right)$ & $\bar{Z}_{n}\left(q_{2} r\right)$ \\
\hline$\omega>a$ & $J_{n}\left(q_{1} r\right)$ & $Y_{n}\left(q_{1} r\right)$ & $J_{n}\left(q_{2} r\right)$ & $Y_{n}\left(q_{2} r\right)$ \\
$a>\omega>b$ & $I_{n}\left(q_{1} r\right)$ & $K_{n}\left(q_{1} r\right)$ & $J_{n}\left(q_{2} r\right)$ & $Y_{n}\left(q_{2} r\right)$ \\
$\omega<b$ & $I_{n}\left(q_{1} r\right)$ & $K_{n}\left(q_{1} r\right)$ & $I_{n}\left(q_{2} r\right)$ & $K_{n}\left(q_{2} r\right)$ \\
\hline If $a=\lambda \sqrt{\frac{E}{[\rho h(1+v)(1-2 v)]}} \& b=\lambda \sqrt{\frac{E}{[2 \rho h(1+v)]}}$
\end{tabular} \\
\hline
\end{tabular}

By substituting equation 6 in equation 1 the stress equation in the surface of cylindrical shells are obtained as follows:

$$
\begin{aligned}
& \sigma_{r}=\frac{E}{(1+v) r^{2}}\{ \\
& {\left[\begin{array}{l}
\frac{1}{2}\left[2 n(n-1)+\left(\lambda^{2}-P_{2}^{2}\right) r^{2}\right] \\
Z_{n}\left(q_{1} r\right)+\zeta q_{1} r Z_{n+1}\left(q_{1} r\right)
\end{array}\right] A_{m n}} \\
& +\left[\begin{array}{l}
\frac{1}{2}\left[2 n(n-1)+\left(\lambda^{2}-P_{2}^{2}\right) r^{2} \bar{Z}_{n}\left(q_{1} r\right)+\right. \\
q_{1} r \bar{Z}_{n+1}\left(q_{1} r\right)
\end{array}\right] \\
& B_{m n}+ \\
& {\left[\left[n(n-1)-P_{2}^{2} r^{2}\right] Z_{n}\left(q_{2} r\right)+\zeta q_{2} r Z_{n+1}\left(q_{2} r\right)\right] C_{m n}+(7)} \\
& {\left[n(n-1)-P_{2}^{2} r^{2}\right] \bar{Z}_{n}\left(q_{2} r\right)+q_{2} r \bar{Z}_{n+1}\left(q_{2} r\right) D_{m n}+} \\
& {\left[n(n-1) Z_{n}\left(q_{2} r\right)-\zeta n q_{2} r Z_{n+1}\left(q_{2} r\right) E_{m n}+\right.} \\
& \left.\left[n(n-1) \bar{Z}_{n}\left(q_{2} r\right)-n q_{2} r \bar{Z}_{n+1}\left(q_{2} r\right)\right] F_{m n}\right\} \\
& \sin \lambda * x \cos n \varphi \cos \omega t
\end{aligned}
$$

Vol. 5 No. 9 (Sep. 2012)

ISSN: 0974- 6846

$$
\begin{aligned}
& \tau_{r \varphi}=\frac{E}{(1+v) r^{2}}\left\{\left[-n(n-1) Z_{n}\left(q_{1} r\right)+\zeta n q_{1} r Z_{n+1}\left(q_{1} r\right)\right] A_{m n}+\right. \\
& {\left[-n(n-1) \bar{Z}_{n}\left(q_{1} r\right)+n q_{1} r \bar{Z}_{n+1}\left(q_{1} r\right)\right] B_{m n}+} \\
& {\left[-n(n-1) Z_{n}\left(q_{2} r\right)+\zeta n q_{2} r Z_{n+1}\left(q_{2} r\right)\right] C_{m n}+\quad(8)} \\
& {\left[(n-1) \bar{Z}_{n}\left(q_{2} r\right)+n q_{2} r \bar{Z}_{n+1}\left(q_{2} r\right) D_{m n}\right]+} \\
& {\left[-\left(n^{2}-n-\frac{P_{2}^{2} r^{2}}{2}\right) Z_{n}\left(q_{2} r\right)-\zeta q_{2} r Z_{n+1}\left(q_{2} r\right) E_{m n}+\right.} \\
& \left.\left[-\left(n^{2}-n-\frac{P_{2}^{2} r^{2}}{2}\right) \bar{Z}_{n}\left(q_{2} r\right)-q_{2} r Z_{n+1}\left(q_{2} r\right)\right] F_{m n}\right\} \sin \lambda * x \sin n \varphi \cos \omega t \\
& \tau_{r \varphi}=\frac{E}{(1+v) r^{2}}\left\{n \lambda r Z_{n}\left(q_{1} r\right) A_{m n}+n \lambda r \bar{Z}_{n}\left(q_{1} r\right) B_{m n}+\right. \\
& \frac{1}{2}\left(-\frac{n P_{2}^{2} r}{\lambda}+n \lambda r\right) Z_{n}\left(q_{2} r\right) C_{m n}+\frac{1}{2}\left(-\frac{n P_{2}^{2} r}{\lambda}+n \lambda r\right) \bar{Z}_{n}\left(q_{2} r\right) D_{m n}+(9) \\
& \frac{1}{2}\left[n \lambda r Z_{n}\left(q_{2} r\right)-\zeta q_{2} r \lambda r Z_{n+1}\left(q_{2} r\right) E_{m n}+\right. \\
& \frac{1}{2}\left[n \lambda r \bar{Z}_{n}\left(q_{2} r\right)-\zeta q_{2} \lambda^{2} r \bar{Z}_{n+1}\left(q_{2} r\right) F_{m n} \cos \lambda * x \sin n \varphi \cos \omega t\right.
\end{aligned}
$$

For above equation we have:

$$
\begin{aligned}
& 1 \text {, if }=\left\{\begin{array}{cc}
J_{n} \& Y_{n} & \text { are Bessel function of type } Z_{n}, \overline{Z_{n}} \\
I_{n} \& K_{n} & \text { are Bessel function of type }
\end{array}\right. \\
& Z_{n}, \overline{Z_{n}} \quad-1 \text {, if }
\end{aligned}
$$

\section{The Frequency Equation}

The equation governing the surface of cylindrical shells should satisfy boundary conditions. By substituting governing boundary condition on inner and outer surface of cylindrical shells, six algebraic equations are obtained for unknown constant Amn, Bmn, Cmn, Dmn, Emn, Fmn which should not be zero. For nontrivial solution, the determinant of algebraic equation must vanish.

$$
\left[\begin{array}{llllll}
a_{11} & a_{12} & a_{13} & a_{14} & a_{15} & a_{16} \\
a_{21} & a_{22} & a_{23} & a_{24} & a_{25} & a_{26} \\
a_{31} & a_{32} & a_{33} & a_{34} & a_{35} & a_{36} \\
a_{41} & a_{42} & a_{43} & a_{44} & a_{45} & a_{46} \\
a_{51} & a_{52} & a_{53} & a_{54} & a_{55} & a_{56} \\
a_{61} & a_{62} & a_{63} & a_{64} & a_{65} & a_{66}
\end{array}\right]=0
$$

\section{Results and discussion}

The numerical results obtained from exact elasticity theory for the frequency parameters $\Omega$ and $\varpi$ for different vibration modes and different proportions $\mathrm{R} / \mathrm{L}$ and $\mathrm{H} / \mathrm{L}$ 
Table 2. Compares frequency parameter $\Omega=\omega H / \pi(\rho / G)^{1 / 2}$ for all method $\mathrm{V}=0.3, m=1, H / R=0.1, R=1$

\begin{tabular}{|l|l|l|l|l|}
\hline $\mathrm{n}$ & $\mathrm{H} / \mathrm{L}$ & $\begin{array}{l}\text { Armenakas } \\
\text { et al. (1969) }\end{array}$ & $\begin{array}{l}\text { Elasticity } \\
\text { (Soldatos \& } \\
\text { Hadjigeorgiou, } \\
1990)\end{array}$ & Exact \\
\hline \multirow{4}{*}{1} & 0.01 & 0.00040 & 0.00041 & 0.000407 \\
\hline 0.1 & 0.03563 & 0.03563 & 0.0356317 \\
\hline 0.2 & 0.11368 & 0.11368 & 0.11368 \\
\hline 0.3 & 0.20451 & 0.20451 & 0.20451 \\
\hline 0.4 & 0.29836 & 0.29836 & 0.298357 \\
\hline 0.6 & 0.48693 & 0.48698 & 0.490021 \\
\hline 0.8 & 0.67583 & 0.67597 & 0.676109 \\
\hline 1.0 & 0.86589 & 0.86618 & 0.86761 \\
\hline 0.01 & 0.65850 & 0.6589 & 0.6587 \\
\hline 0.1 & 0.65957 & 0.65997 & 0.66004 \\
\hline 0.2 & 0.66496 & 0.66535 & 0.66729 \\
\hline 0.3 & 0.67876 & 0.67914 & 0.684893 \\
\hline 0.4 & 0.70383 & 0.70421 & 0.71284 \\
\hline 0.6 & 0.78912 & 0.78952 & 0.791021 \\
\hline 0.8 & 0.91240 & 0.91285 & 0.914049 \\
\hline 1.0 & 1.06030 & 1.06084 & 1.06084 \\
\hline
\end{tabular}

have been compared with the results that have been already obtained by other methods. Table 2 , shows the comparison of frequency parameter of $\Omega=\omega \mathrm{H} / \pi(\rho / \mathrm{G}) 1 / 2$ with the results of Armenakas et al., 1969 and Loy \& Lam (1999) for cylindrical shells with 25 layers:

Table. 3 Compares frequency parameter $\Omega=\omega H / \pi(\rho / G) 1 / 2$ for all method $v=0.3, m=1, H / R=1.0, R=1$

\begin{tabular}{|l|l|l|l|}
\hline $\mathrm{Hh} / \mathrm{L}$ & $\begin{array}{l}\text { Armenakas } \text { et } \\
\text { al. }(1969)\end{array}$ & $\begin{array}{l}\text { Loy \& Lam } \\
(1999)\end{array}$ & Exact \\
\hline 0.01 & 0.00023 & 0.00058 & 0.005746 \\
\hline 0.1 & 0.02423 & 0.02434 & 0.022451 \\
\hline 0.2 & 0.08703 & 0.08709 & 0.08701 \\
\hline 0.4 & 0.26658 & 0.26659 & 0.26739 \\
\hline 0.6 & 0.46827 & 0.468256 & 0.470917 \\
\hline 0.8 & 0.67316 & 0.67316 & 0.67772 \\
\hline 1.0 & 0.87660 & 0.87665 & 0.8835 \\
\hline
\end{tabular}

Table.3 show the results of frequency parameters $\Omega$ $(\Omega=\omega H / \quad \pi(\rho / G) \quad 1 / 2)$ for $H / R=0.1$ and different $H / L$ proportions. Table 2 shows that, when proportion of $\mathrm{H} / \mathrm{L}$ increases, amount of agreement between the results increasing. That means when the length of cylindrical shell.

Table.4 compares the results obtained from exact elasticity theory with other available for frequency parameters $\varpi\left(\varpi=L \omega \sqrt{\frac{\rho}{E}(1+v)}\right)$ the results for different proportions of $\mathrm{H} / \mathrm{Land}$ different vibrations have been display.

Conclusion

This study was about vibration of isotropic cylindrical shells on the basis of exact elasticity theory for simple supported boundary condition. The results were compared whit the results that have already been obtained through other theories. The results have been obtained on the basis of different proportions of $H / L$ and $L / R$ and different vibration modes and have been compared. We can see
Table 4. Compares frequency parameter $\varpi$ for all method $\mathrm{v}=0.3$, $m=1, H / R=1.0, R=1$

\begin{tabular}{|l|l|l|l|l|l|}
\hline $\mathrm{H} / \mathrm{L}$ & $\mathrm{n}$ & $\begin{array}{l}\text { Soldatos \& } \\
\text { Hadjigeorgiou } \\
(1990)\end{array}$ & $\begin{array}{l}\text { Armenakas } \\
\text { et al. } \\
(1969)\end{array}$ & $\begin{array}{l}\text { Loy \& Lam } \\
(1999)\end{array}$ & Exact \\
\hline \multirow{6}{*}{0.1} & 1 & 1.06238 & 1.06226 & 1.06225 & 1.06247 \\
& 2 & 0.8826 & 0.88233 & 0.88643 & 0.88229 \\
& 3 & 0.80963 & 0.80925 & 0.80949 & 0.80945 \\
& 4 & 0.89905 & 0.89877 & 0.89880 & 0.89896 \\
& 5 & 1.12216 & & 1.122009 & 1.122378 \\
\hline \multirow{5}{*}{0.2} & 1 & 1.18908 & 1.18889 & 1.18900 & 1.189106 \\
& 2 & 1.10121 & 1.10092 & 1.10112 & 1.101402 \\
& 3 & 1.19793 & 1.19755 & 1.19777 & 1.19807 \\
& 4 & 1.48975 & 1.48933 & 1.48971 & 1.50138 \\
& 5 & 1.91389 & & 1.91406 & 1.21088 \\
\hline \multirow{5}{*}{0.3} & 1 & 1.33761 & 1.33727 & 1.33748 & 1.33701 \\
& 2 & 1.32371 & 1.32335 & 1.32359 & 1.33694 \\
& 3 & 1.52805 & 1.52764 & 1.52804 & 1.53208 \\
& 4 & 1.92695 & 1.92660 & 1.92722 & 1.93157 \\
& 5 & 2.44628 & & 2.44692 & 2.45105 \\
\hline
\end{tabular}

that the difference between results have never been more than $10 \%$ of vibration modes increase, where this difference decreases. In general there is a good agreement between the results of all of the theories.

\section{References}

1. Alibeigloo A and Shakeri M (2007) Elasticity solution for the free vibration analysis of Laminated cylindrical panels using the differential quadrature method. Composite Structures. 81 (1), 105-113.

2. Armenakas AE, Gazis Ds and Herrman G (1969) Free vibration of circular cylindrical shell. Oxford: Pergamon Press.

3. Flügge JW (1993) Stresses in shell. Berlin: Springer.

4. Love AEH (1995) Reatise on the mathematical theory of elasticity. Phil. Trans. Roy. Soc. London. A247, 529551.

5. Loy CT and Lam KY (1999) Vibration of thick cylndrical shells on the basis of three-densional theory of elacticity. J. Sound Vib. 226, 719-737

6. Soldatos KP and Hadjigeorgiou VP (1990) Three dimension solution of the free vibration problem of homogeneous isotropic cylindrical shells and panels. J. Sound \& Vibr. 137, 369-384.

7. Stefan Markus(1989) The mechanics of vibrations of cylindrical shells, Sevier. Amsterdam-Oxford-New York-Tokyo Sci.

8. Timoshenko SP (1989) Theory of plates and shells. Mc Graw-Hill, Singapore. 